\title{
Detection Method of Meso-Scale Micro-Damage Based on Image Processing Technique
}

\author{
Xiaohui $\mathrm{Ni}^{1,2, a}$ \\ ${ }^{1}$ College of Civil Engineering \& Architecture, Jiaxing University, Jiaxing,Zhejiang, 314001,China \\ ${ }^{2}$ Department of Civil Engineering, Shaoxing University, Shaoxing, Zhejiang 312000, P.R.China \\ axhnmeeting@163.com
}

Keywords: Rock mechanics, Meso Scale, Meso crack, Marble.

Abstract. Basded on 2D and 3D geometry theories in stereology, there was the method of acquisition of three-dimensional geometric information parameters. On this basis of this method, with meso scale of microcracks for the marble, there was the result that the meso-scale marble microcracks spacing varying parameters during uniaxial compression obeyed generalized limit distribution of this feature. And there was a range of energy threshold during the development of microcracks.

\section{Introduction}

The rock is the product in a long geological movement. It is mixed body of a variety of mineral grains and cement composition. There are a lot of microcracks in different number and scales. This has resulted in more complex features in the chemical composition and physical properties. There are diversity, nonlinear, time-varying anisotropy, and rheological properties for its mechanical properties. Therefore, it is the hot topics for the current international civil engineering to study meso-scale mechanism of injury. So the further research meso-scale damage mechanism is very important for theoretical and practical significance.

In recent years, for the rapid development of structural analysis basing on various types of electron microscopy test technology, scholars began to use various types of scanning electron microscopy meso-scale analysis for micro-damage measure.Michael et al. [1] considered that only based a multi-scale mechanical model the principle of the mechanism of meso-crack initiation should be investigated. Brittle or ductile materials should be studied on the meso-level. There are lots of the initial micro-cracks and micro-defects in rock due to the particularity of the rock diagenetic process. This is the initial damage in the concept of damage mechanics. The fracture failure of rock is caused by the expansion, merge and through of micro-cracks and micro-defects and finally macro-cracks forming under load. Therefore, the degree of initial damage of the rock have a negligible effect on the mechanical properties.Compell et al.[2] discussed the feasibility of using an optical microscope to quantify the micro-structure of some materials such as rocks, bricks and cements. They compared the method with that of X-ray. Robina et al.[3] studied how the initial micro-crack density and micro-particles scale impact on the uniaxial compressive strength of marble samples. In 1997, Yossef et al.[4] studied the relationship between the micro-structure of dolomite, the initial stress of micro-crack initiation and sample's ultimate strength. He found that the micro-structure have a big impact on the ultimate strength. Menendez et al.[5] discussed the approach of using a con-focal laser scanning microscope to study the micro-cracks and micro-pore network of rocks. But for the restrictions by the image information processing technology, most of the studies were staying on qualitative research level. It does not meet the needs of quantitative levels of mechanics. As the development of the iconography and digital image processing techniques, it is possible to obtain meso- and micro-damage information from the SEM image of rocks.

On this basis, this study was combined with Matlab image processing software package to establish the meso-scale micro-damage recognition method and to write associated image processing program, which can accurately obtain meso-structure basic geometric data. It is significant for further meso quantitative study for micro-scale damage of rokcs. And it is helpful to study rocks from the perspective of multi-scale damage failure process. 


\section{The acquisition principle for meso-scale damage geometrical parameter of rock}

Constitution of rock. There are four kinds of basic meso-scale microstructure unit in rock: (1) It is three-dimensional structure, such as the meso-scale of mineral particles, micro-cracks;(2) It is two-dimensional surface of structure, including the meso-scale of mineral particles, cemented face surface and a variety of mineral particles between microcracks; (3) It is a one-dimensional line, including all kinds of micro-meso scale intergranular mineral particles transgranular microcracks, mineral particles and the cemented surface between the cracks;(4) It is zero-dimensional points, including microscopic scale fine micro cracks, holes and so on. And we concentrated on meso geometric characteristics of the micro-structural damage, including the volume of the three-dimensional structure, the area of two-dimensional structure, the length and curvature of one-dimensional line. From the viewpoint of surveying, based on a variety of sample analysis and calculation on measuring devices, we can get meso structural damage geometric properties of rock.

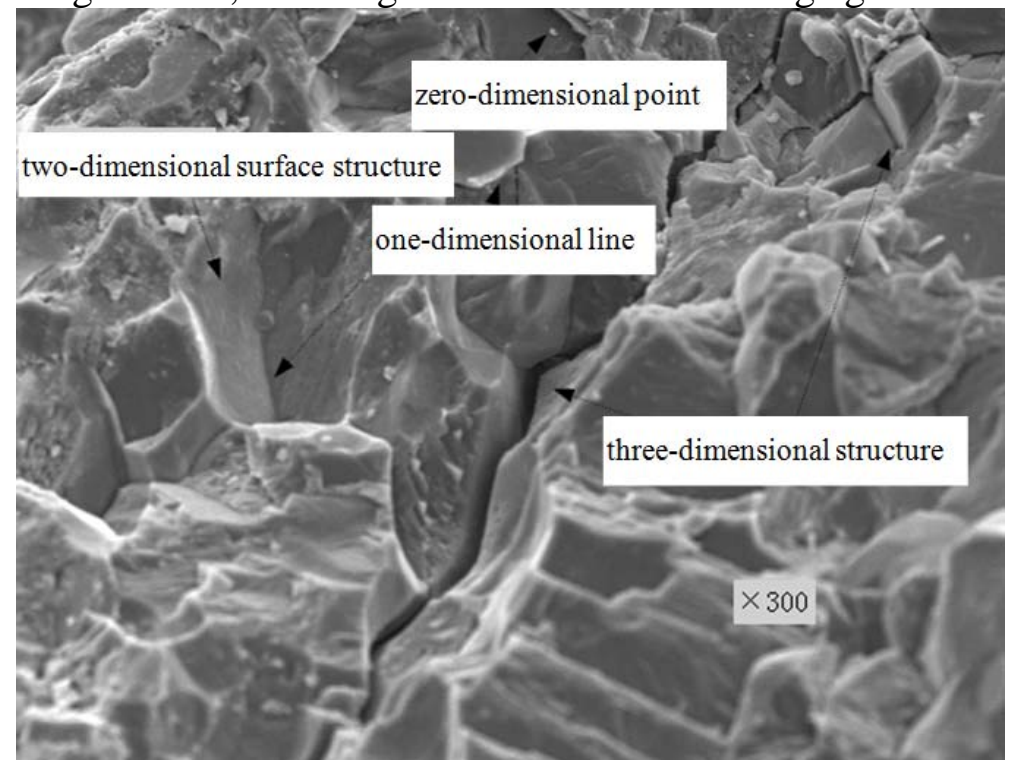

Fig.1 Flowchart of preparation of specimen

Rock meso-structure SEM images processing program. In recent years, with the development of computer technology and digital image processing technology, it becomes feasible that the characteristic information of rock meso-structure is quantified with the analysis of meso-image of rock samples. With the development of digital image processing technology, it is easier, more accurate, more representative in statistics to obtain data collecting from meso-image of samples. The datamation of image of rock microcracks by SEM belongs to the module of image analysis treatment in digital image processing. During of the process of formation, transmission and recording of the SEM images, the SEM image quality, such as blurred image, deformation image, etc., declined for many factors, which were the image error of optical system, the relative motion between imaging equipment and objects and the distortion during image processing. Based on characteristics of rock meso-structure SEM images, the region growing method is used for image segmentation, and the relative image recognition program is compiled by combining image processing toolbox in Matlab, so SEM image data is obtained accurately ( Fig.2 ). 


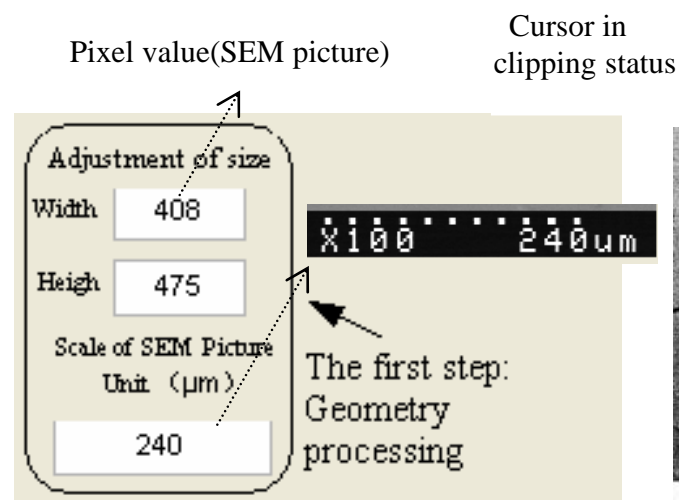

(b) Adjustment of size

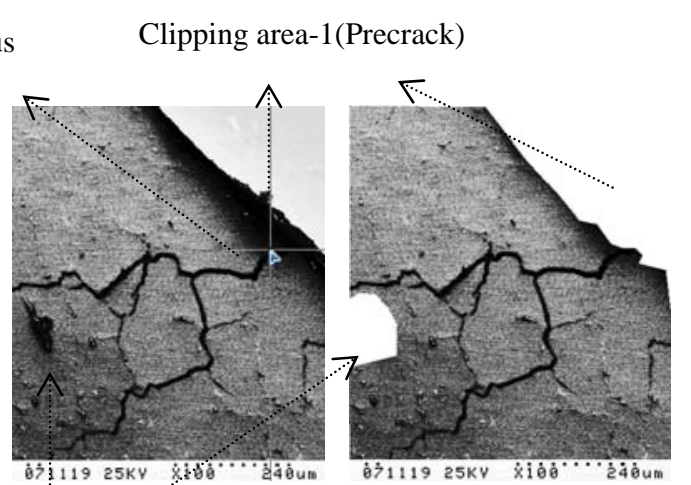

(c) Clipping algorithm

Clipping area-2(Impurities)

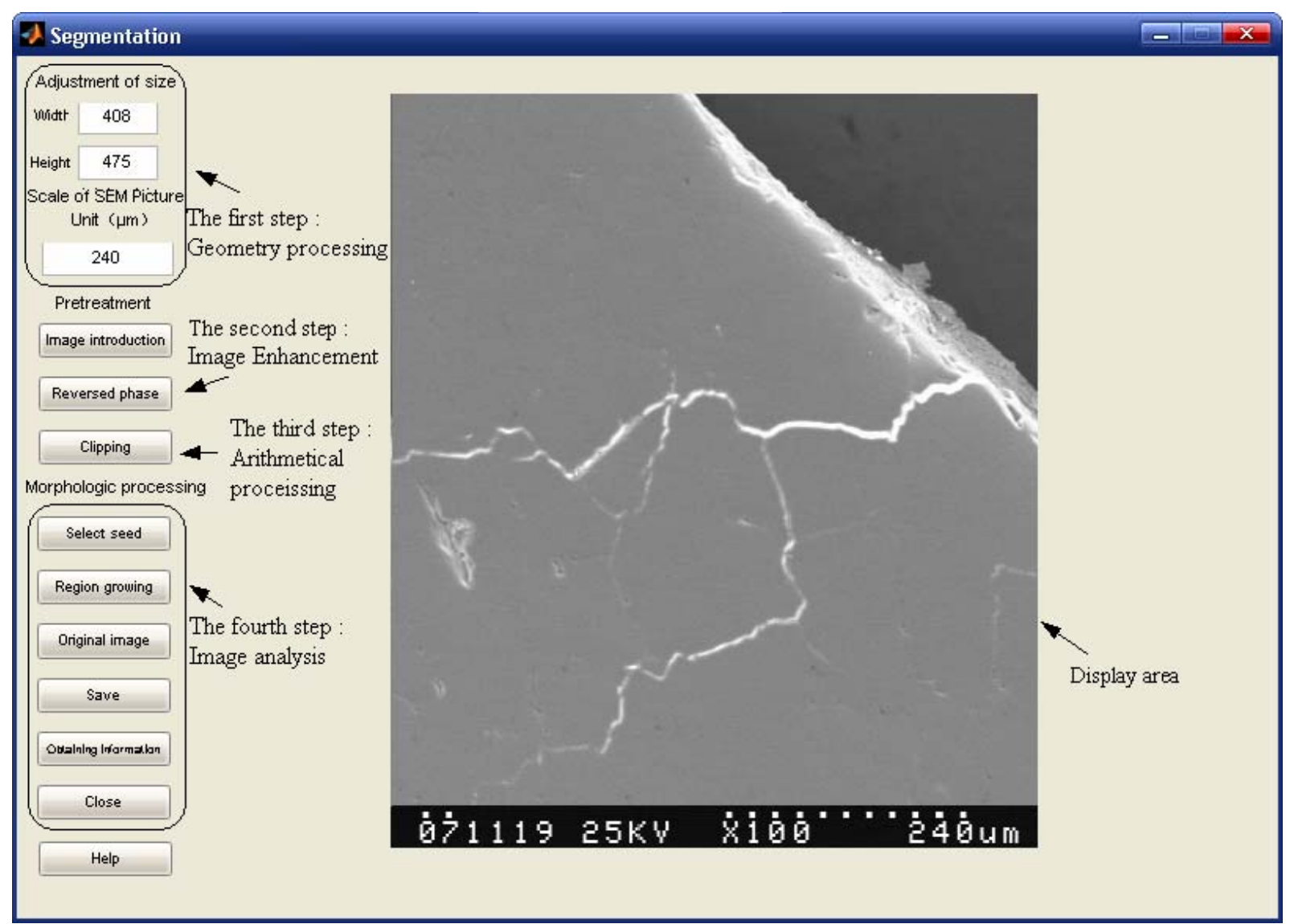

(a) Operation of image processing program

Seed (The white point) Microcracks
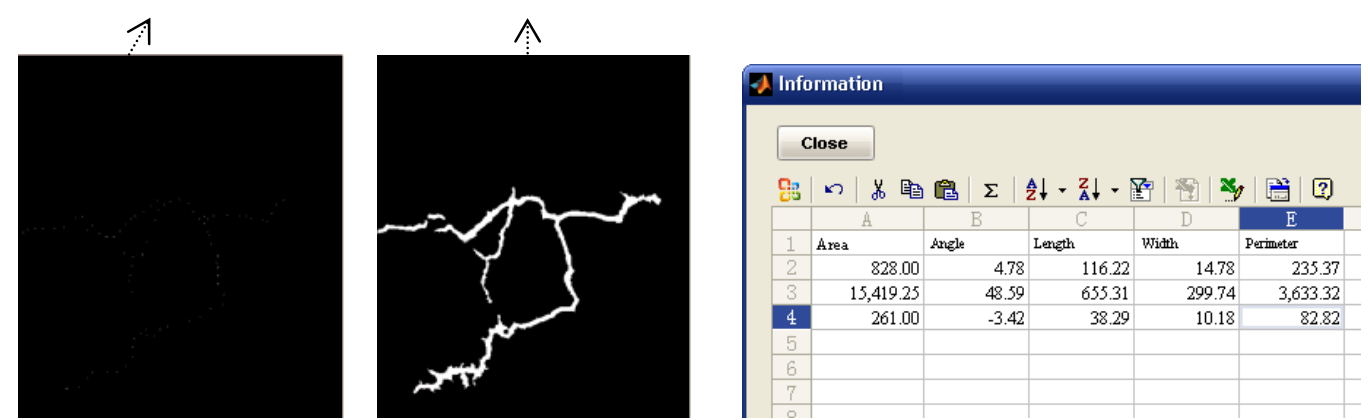

(d) Image Segmentation of Regional growing (e) Statistical data of the image Fig3 Introduction of the program for datamation of image 


\section{Conclusions}

(1) Based on three-dimensional geometric parameters of stereological geometry, the relationship between two-dimensional and three-dimensional geometric features geometric characteristics was derived from the microcracks of marble SEM image.

(2) A SEM images processing program for rock meso-structure is compiled to process rock microcracks SEM image. Thus, the parameters such as the microcrack length, azimuth angle, width, area and perimeter, etc. can be extracted from microcrack structure under different stress state.

\section{Acknowledgements}

This work was financially supported by the Natural Science Foundations of Zhejiang Province (No. LQ12E08004).

\section{References}

[1] Michael E. Kassner, Sia Nemat-Nasser, Zhigang Suo, et al: New Directions in Mechanics. Mechanics of Materials, Vol. 37(2005), p. 231-259.

[2] Campbell, Donald H, Galehouse et al.: Quantitative Clinker Microscopy with the Light Microscope. Cement, Concrete and Aggregates, Vol. 13(1991) , p. 94-96.

[3] Robina H C, Wong K T, Chau P Wang.: Microcracking and Grain Size Effect in Yuen Long Marbles. Int. J. Rock Mech. Min.Sci. \& Geomech. Abstract, Vol. 33(1996) , p. 479-485.

[4] Yossef H Hatzor, Alon Zur, Yaakov Mimran.: Microstructure Effects on Microcracking and Brittle Failure of Dolomites. Tectonophysics, Vol. 281(1997) , p. 141-161

[5] Menendez B, David C, Nistal A M.: Confocal Scanning Laser Microscopy Applied to the Study of Pore and Crack Networks in Rocks. Computers and Geosciences, Vol. 27(2001) , p. 1101-1109 